\title{
Fever and Leg Pain: Consider ALL the Diagnoses
}

\author{
Lesley N. Desmond, MD, ${ }^{1}$ Matthew B. Fletcher, MD, ${ }^{2}$ Rajasekharan P. Warrier, MD $^{1,2}$ \\ ${ }^{1}$ The University of Queensland Faculty of Medicine, Ochsner Clinical School, New Orleans, LA ${ }^{2}$ Department of Pediatrics, Ochsner Clinic \\ Foundation, New Orleans, LA
}

Background: Acute lymphoblastic leukemia (ALL) is the most common childhood cancer diagnosed in the United States. The disease causes a decrease in hematopoiesis, so children often present with symptoms related to anemia, thrombocytopenia, and leukopenia. Symptoms for this malignancy may have significant overlap with other conditions such as osteomyelitis.

Case Report: A 2-year-old male with no significant medical history presented with lower extremity pain and fever. Initial investigations, including imaging and complete blood count, led physicians to diagnose bilateral osteomyelitis. The patient was prescribed a course of antibiotics; however, his symptoms returned. Eventually, a bone marrow aspiration showed CD99 membrane-positive small round blue cell tumors. The patient was diagnosed with ALL. He was successfully treated with chemotherapy and is now in remission.

Conclusion: This case demonstrates the importance of a broad differential diagnosis for a child presenting with leg pain and fever.

Keywords: Anemia, bone marrow, chemotherapy, erythrocyte sedimentation rate, fever, leukocyte counts, leukopenia, osteomyelitis, precursor B-cell lymphoblastic leukemia-lymphoma, thrombocytopenia

Address correspondence to Rajasekharan P. Warrier, MD, Department of Pediatrics, Ochsner Clinic Foundation, 1514 Jefferson Hwy., New Orleans, LA 70121. Tel: (504) 842-3900. Email: rwarrier@ochsner.org

\section{INTRODUCTION}

Acute lymphoblastic leukemia (ALL) is the most common childhood cancer diagnosed in the United States. ${ }^{1}$ The disease causes a decrease in hematopoiesis, so children often present with symptoms related to anemia, thrombocytopenia, and leukopenia. ${ }^{2}$ The classic triad of easy bruising, lethargy, and fever is present in only approximately one-quarter of patients. Other typical symptoms include musculoskeletal pains (especially leg pain), anorexia, and abdominal pain. Common physical examination findings, in addition to purpura, fever, and pallor, are hepatomegaly, splenomegaly, and lymphadenopathy. ${ }^{2}$

We present the case of a patient with suspected bilateral osteomyelitis that demonstrates the need to consider malignancy and perform a workup for leukemia in children who present with persistent bone pain and fever.

\section{CASE REPORT}

A previously healthy 2-year-old Caucasian male presented to the emergency department with swelling of the left foot. His adoptive parents noticed the swelling after his foot was caught in the slats of his crib. Physical examination was negative for deformity and swelling of the limb, rash, pallor, fever, and organomegaly. Left foot and ankle x-rays showed no fracture or dislocation. The patient was discharged home to be followed by his pediatrician.

Nine days later, the patient presented to his pediatrician with fever and refusal to bear weight on his lower limbs. Review of systems revealed chills and decreased appetite in addition to fever and leg pain. Physical examination showed tenderness and warmth on both ankles but no erythema or swelling and was negative otherwise. Initial laboratory studies were notable for a low white blood cell count of $4.27 \mathrm{~K} / \mu \mathrm{L}$ (reference range, $6.00-17.50 \mathrm{~K} / \mu \mathrm{L}$ ), hemoglobin of $11.1 \mathrm{~g} / \mathrm{dL}$ (reference range, $10.5-13.5 \mathrm{~g} / \mathrm{dL}$ ), low hematocrit of $32.4 \%$ (reference range, $33.0 \%-39.0 \%$ ), high platelets of $377 \mathrm{~K} / \mu \mathrm{L}$ (reference range, $150-350 \mathrm{~K} / \mu \mathrm{L}$ ), and an erythrocyte sedimentation rate (ESR) of $71 \mathrm{~mm} / \mathrm{hr}$ (reference range, 0-10 mm/hr). The leukocyte differential was within normal limits other than a slightly increased percentage of lymphocytes. Absolute neutrophil count was within the reference range.

The patient returned to his pediatrician 2 days later for left ankle pain. White blood cell count, hemoglobin, and hematocrit were all below reference ranges. His ESR was further elevated to $95 \mathrm{~mm} / \mathrm{hr}$. Lower extremity x-rays showed no fracture, dislocation, or bone destruction. After orthopedic evaluation, the patient was admitted to the hospital for 5 days with concern for osteomyelitis, cellulitis, or septic arthritis.

$\mathrm{He}$ was started on intravenous (IV) clindamycin $40 \mathrm{mg} /$ $\mathrm{kg} /$ day divided into 3 doses given every 8 hours for 2 days. In addition to antibiotic therapy, the patient was prescribed oral ibuprofen $10 \mathrm{mg} / \mathrm{kg}$ as needed every 6 hours. Magnetic resonance imaging (MRI) revealed areas of bone marrow signal hypointensity with peripheral enhancement of the left distal tibia and left distal fibula. Periostitis and the possibility of developing intraosseous abscesses were considered 


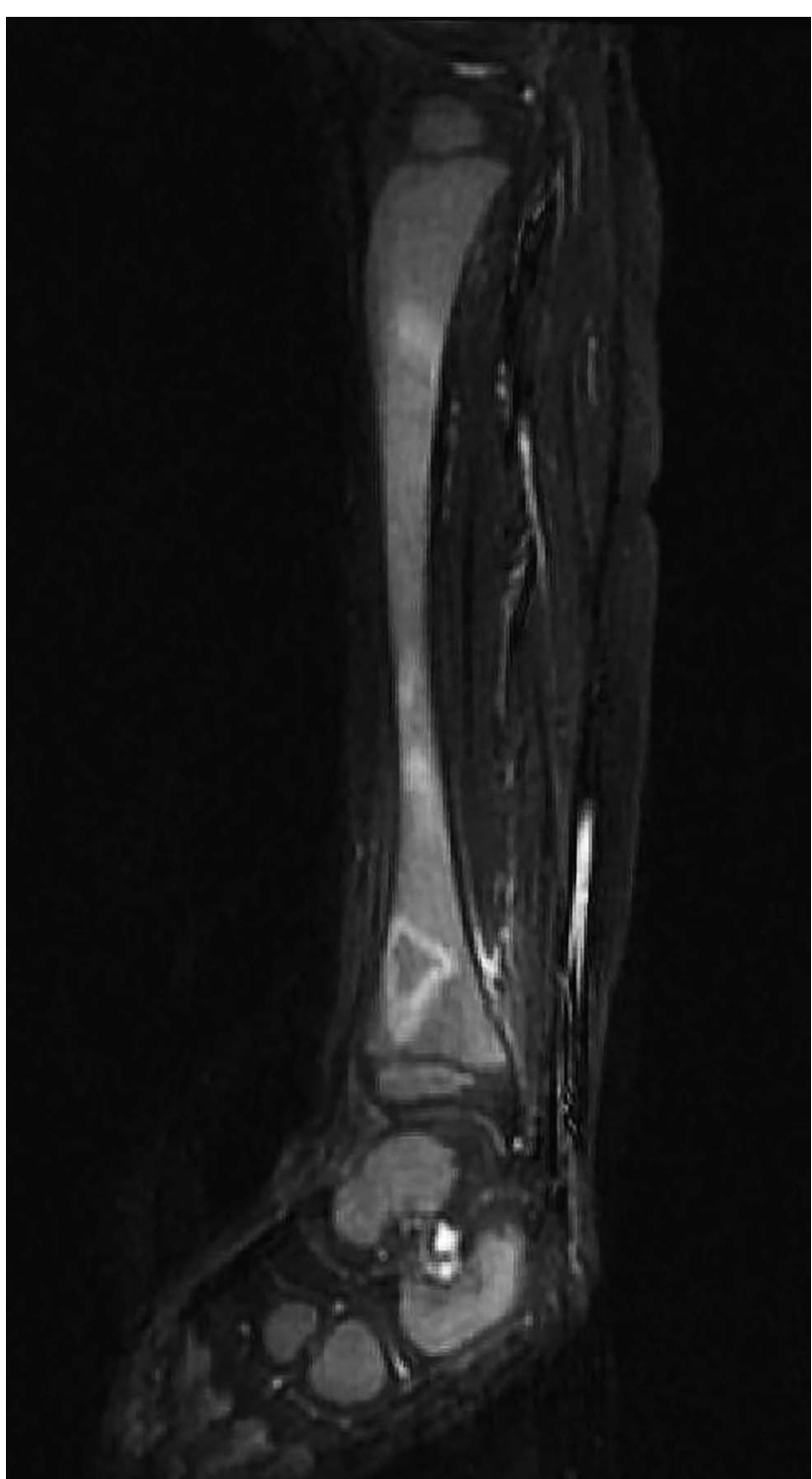

Figure. Magnetic resonance imaging (MRI) of the right leg shows a $1.1 \times 0.6 \times 2.4 \mathrm{~cm}$ focal area of serpiginous abnormal bone marrow signal in the distal tibial metaphysis. The area demonstrates an enhancing, hyperintense peripheral rim and central nonenhancement. MRI of the left leg showed similar findings.

possibilities. C-reactive protein (CRP) was elevated at $45.3 \mathrm{mg} / \mathrm{L}$ (reference range, 0.0-8.2 mg/L). Blood cultures showed no growth after 5 days, and CRP decreased to $10.8 \mathrm{mg} / \mathrm{L}$. The patient was afebrile and able to ambulate without pain, and he was discharged home on oral clindamycin $40 \mathrm{mg} / \mathrm{kg} /$ day for a total of 21 days.

On day 17 of antibiotics, the patient returned for followup with his pediatrician. He had a fever, warmth and redness of the right foot, and a limp. He was readmitted for 10 days. X-rays showed periosteal thickening of both the tibias and fibulas bilaterally, consistent with possible bilateral osteomyelitis. MRI of the lower extremities demon- strated mild periostitis and abnormal bone marrow signal concerning for osteomyelitis with possible early developing intraosseous abscesses (Figure), similar to abnormalities shown on the MRI from the previous hospitalization. Peripheral rim enhancement with central nonenhancement was noted, along with persistent heterogeneous bone marrow signal.

After 2 days of hospitalization, curettage samples of the affected bones were sent for pathologic examination. Initial testing showed CD99 membrane-positive small round blue cell tumors bilaterally. The differential diagnoses for this bilateral malignancy included Ewing sarcoma, primitive neuroectodermal tumor, undifferentiated sarcoma, hematopoietic lymphoblastic tumor, and other metastatic or primary small blue cell tumor. Computed tomography (CT) scan of the chest, abdomen, and pelvis showed no evidence of metastatic disease. Microscopy showed a highly atypical infiltrate, and the cells were positive for CD34, terminal deoxynucleotidyl transferase (TdT), CD10, sporadic CD20, and CD99 by immunohistochemical staining. The tumor was comprised of sheets of immature B cells in a background of osteonecrosis, sclerosis, and new bone formation that could be consistent with either precursor B-cell lymphoblastic lymphoma or precursor B-cell ALL. Positron emission tomography/CT scan showed no abnormalities aside from the previously noted changes in the lower extremities. Right and left iliac crest bone marrow biopsies showed atypical immature lymphoid cells, some of which demonstrated hand-mirror morphology. Diagnosis of precursor B-cell ALL was confirmed with $52 \%$ blasts in the bone marrow from the right iliac crest and $86 \%$ from the left. Further analysis by immunophenotyping revealed it to be a case of precursor B-cell ALL.

Induction chemotherapy was initiated per the Children's Oncology Group AALL0932 protocol. $^{3}$ The patient achieved complete remission after the first month of induction therapy and subsequently received consolidation therapy, augmented interim maintenance therapy, and augmented delayed intensification therapy. He received maintenance therapy for 3 years and is in continued remission.

\section{DISCUSSION}

More than 3,000 new cases of ALL are diagnosed in the United States each year. The highest rates of ALL are for children 1-4 years old. Males and Hispanics have higher rates of ALL diagnosis than females and other ethnic groups. Interestingly, rates of ALL tend to be higher in the western United States. ${ }^{1}$

As noted previously, ALL patients present with symptoms attributable to the reduction of hematopoiesis. Anemia presents as pallor and lethargy, leukopenia causes infection, and cytokines lead to fever. Our patient presented with limb pain and fever only, so he was among the three-quarters of patients who do not present with the classic ALL symptom triad of easy bruising, lethargy, and fever.

The initial workup for patients suspected to have ALL includes a complete blood count (CBC) that will often show anemia, thrombocytopenia, or both. CBC is often helpful in diagnosis. However, CBC may not reveal any abnormalities, in which case a strong suspicion because of bone lesions or other findings may lead to a bone marrow biopsy showing 
ALL. Measurement of procalcitonin can help to differentiate osteomyelitis from malignancies such as leukemia. Procalcitonin is generally normal in malignancy and increased in infection. ${ }^{4}$ Leukocyte counts are variable. In approximately one-quarter of patients with B-cell ALL, the leukocyte count is $<5 \mathrm{~K} / \mu \mathrm{L}$, but approximately one-half of patients with acute leukemia have a count $>20 \mathrm{~K} / \mu \mathrm{L} .^{5}$

The differential diagnoses for limb pain with fever are quite broad. Considerations include osteomyelitis, lymphoma, metastatic malignancy, septic arthritis, primary bone malignancy such as Ewing sarcoma or osteosarcoma, autoimmune diseases such as juvenile idiopathic arthritis, reactive arthritis, neuroblastoma, and transient synovitis. Fever and bone pain are obviously not specific for osteomyelitis.

Children with ALL commonly have imaging abnormalities. For example, our patient presented with lower extremity pain that needed to be evaluated. CT and MRI can be used to assess patients with suspected malignancy. The most common changes are metaphyseal lucencies. Although not pathognomonic, metaphyseal lucencies are reported to be present in up to $70 \%$ of childhood ALL diagnoses. ${ }^{6}$ These lucencies are most commonly found in the rapidly growing long bones and present in the wrists, knees, or as in our patient, the ankles. Other lesions seen at the onset of ALL include osteolytic lesions, osteopenia, periosteal reaction, and fracture. Periosteal reaction, also known as periostitis, was seen in our patient. This process occurs when new bone is formed following leukemic infiltration and appears as an elevation of the periosteum. ${ }^{7}$ Periostitis can also be seen in acute osteomyelitis along with bone marrow edema and intraosseous abscesses that appear as hypointense lesions on MRI. While MRI has a high sensitivity for osteomyelitis, its specificity is low, and the osteomyelitis can look similar to neuropathic arthropathy, trauma, and malignancy. Two characteristics can help distinguish malignancy from osteomyelitis. First, malignancy tends to show slower destruction on serial MRIs compared to osteomyelitis. Second, abscesses show peripheral rim enhancement while malignancies have heterogeneous enhancement. ${ }^{8}$ In addition, as was the case with our patient, bilateral involvement and the lack of a soft tissue component should prompt the consideration of diagnoses other than osteomyelitis, including malignancy.

ALL is definitively diagnosed based on cell morphology and immunophenotyping of bone marrow samples. The presence of $\geq 25 \%$ blasts in the bone marrow is often used to distinguish ALL from acute lymphoblastic lymphoma. ${ }^{9}$

Treatment of ALL begins with remission induction. Remission is both the eradication of malignant cells and the restoration of normal bone marrow function. Remission induction generally involves 4 weeks of combination chemotherapy with vincristine, a steroid such as dexamethasone, and methotrexate. Induction with this combination achieves remission in approximately $95 \%$ of cases. Maintenance therapy with a lower dosage of the same medications given during induction is continued for up to 3 years. If a patient relapses, high-dose chemotherapy with total body irradiation and bone marrow transplantation are used. ${ }^{10}$

In the United States, the prognosis for children diagnosed with ALL is relatively good. In a study of 17,500 children diagnosed with ALL between the years 2001 and 2009, the survival rate at 1 year was $95 \%$, although at 3 years this number fell to $90 \%$, and the 5 -year survival rate was $86 \%$. $^{11}$ These rates improved by $1 \%-2 \%$ if the child was diagnosed between 2004 and 2009 vs 2001 to 2003. Race affected these numbers as well. Black children had both lower 5-year survival rates and less improvement based upon the year diagnosed compared to white American children. ${ }^{11}$ Other variables that confer a worse prognosis for ALL include age $>10$ years, higher white cell count at diagnosis, male sex, presence of central nervous system disease, and T-cell ALL. $^{2}$

\section{CONCLUSION}

This case demonstrates the importance of a broad differential when evaluating a child with fever and limb pain. Leukemia should be a consideration in any child with persistent bone pain and fever, particularly if the symptoms are associated with fatigue, bruising, and bleeding. Careful examination of the smear and sometimes a bone marrow aspiration may aid in early diagnosis.

\section{ACKNOWLEDGMENTS}

The authors have no financial or proprietary interest in the subject matter of this article.

\section{REFERENCES}

1. Siegel DA, Henley SJ, Li J, Pollack LA, Van Dyne EA, White A. Rates and trends of pediatric acute lymphoblastic leukemia United States, 2001-2014. MMWR Morb Mortal Wkly Rep. 2017 Sep 15;66(36):950-954. doi: 10.15585/mmwr.mm6636a3.

2. Moppett J, Dommett R. Clinical presentation and prognostic factors. In: Vora A, eds. Childhood Acute Lymphoblastic Leukemia. Cham, Switzerland: Springer International Publishing; 2017.

3. Woo K, Angiolillo A. AALL0932: Treatment of patients with newly diagnosed standard risk $b=$ lymphoblastic leukemia or localized b-lineage lymphoblastic lymphoma. Children's Oncology Group. www.childrensoncologygroup.org/aall0932. Published November 18, 2015. Updated October 17, 2018. Accessed March 25, 2019.

4. Lee $\mathrm{H}$. Procalcitonin as a biomarker of infectious diseases. Korean J Intern Med. 2013 May;28(3):285-291. doi: 10.3904/kjim.2013.28.3.285.

5. Kilburn LB, Siegel SE, Steuber CP. Clinical assessment and differential diagnosis of the child with suspected cancer. In: Pizzo PA, Poplack DG, eds. Principles and Practice of Pediatric Oncology. 6th ed. Philadelphia, PA: Wolters Kluwer/Lippincott Williams \& Wilkins Health; 2011:132-133.

6. Baty JM, Vogt EC. Bone changes of leukemia in children. Am J Roentgenol. 1935;34:310-313.

7. Mostoufi-Moab S, Halton J. Bone morbiditiy in childhood leukemia: epidemiology, mechanism, diagnosis, and treatment. Curr Osteoporos Rep. 2014 Sep;12(3):300-312. doi: 10.1007/s11914-014-0222-3.

8. Lee YJ, Sadigh S, Mankad K, Kapse N, Rajeswaran G. The imaging of osteomyelitis. Quant Imaging Med Surg. 2016 Apr;6(2):184-198. doi: 10.21037/qims.2016.04.01.

9. Metzeler K. Diagnostic criteria, classification, and prognosis of acute leukemias. In: Hiddemann W, ed. Handbook of Acute Leukemia. Cham, Switzerland: Springer International Publishing; 2016: 25-40. 
10. Lissauer T, Clayden G. Illustrated Textbook of Paediatrics. 5th ed. Edinburgh, UK: Mosby Elsevier; 2018:390-391.

11. Tai EW, Ward KC, Bonaventure A, Siegel DA, Coleman MP. Survival among children diagnosed with acute lymphoblastic leukemia in the United States, by race and age, 2001 to 2009: findings from the CONCORD-2 study. Cancer. 2017 Dec 15;123 Suppl 24;5178-5189. doi: 10.1002/cncr.30899.

This article meets the Accreditation Council for Graduate Medical Education and the American Board of Medical Specialties Maintenance of Certification competencies for Patient Care and Medical Knowledge. 